Fourier state of a fluidized granular gas

This content has been downloaded from IOPscience. Please scroll down to see the full text. 2001 Europhys. Lett. 53432

(http://iopscience.iop.org/0295-5075/53/4/432)

View the table of contents for this issue, or go to the journal homepage for more

Download details:

IP Address: 150.214.182.194

This content was downloaded on 15/06/2015 at 17:28

Please note that terms and conditions apply. 
Europhys. Lett., 53 (4), pp. 432-437 (2001)

\title{
Fourier state of a fluidized granular gas
}

\author{
J. J. Brey, D. Cubero, F. Moreno and M. J. Ruiz-Montero \\ Física Teórica, Universidad de Sevilla - Apartado de Correos 1065 \\ E-41080, Sevilla, Spain
}

(received 22 September 2000; accepted in final form 1 December 2000)

PACS. 05.20.-y - Classical statistical mechanics.

PACS. 05.20.Dd - Kinetic theory.

PACS. 81.05.Rm - Porous materials; granular materials.

\begin{abstract}
It is shown that the Boltzmann equation for smooth inelastic hard disks or spheres admits a solution describing a steady state characterized by uniform pressure and linear temperature profile. Such a state has been observed previously both in numerical solutions of the Boltzmann equation and in molecular dynamics simulations. Quite peculiarly, pressure and temperature gradient are not independent but their ratio is a function of the coefficient of restitution. Several properties of the solution are discussed. In particular, it is shown that a linear Fourier-like law is verified for arbitrary temperature gradient.
\end{abstract}

The Boltzmann equation, modified to account for inelastic two-particle collisions, is often used as an idealized description of dilute rapid granular flows [1]. Nevertheless, very little is known about its solutions. Even for the simplest possible state, corresponding to a freely evolving homogeneous system, the velocity distribution is only known in some approximation [2]. For inhomogeneous situations, the available solutions are restricted to systems with small spatial gradients or to the quasielastic limit [3-5].

Most of the experimental situations deal with a granular system to which energy is continuously supplied in order to keep it fluidized and, eventually, in a steady state. Often, this is done by means of a vibrating wall. The steady state reached by the system is far from equilibrium and also from the time-dependent homogeneous cooling state (HCS) describing the free evolution of a granular system. Except in the limit of very small inelasticity, a direct extension of the hydrodynamic description obtained by expanding around the HCS is not expected to apply. In fact, it is known that inelasticity and gradients are coupled for a granular gas in the presence of a wall providing energy to the system [6,7].

Although the boundary layer next to the vibrating wall must be taken into account for a complete description of the system, far away from the wall the state of the granular flow may be characterized in a simple way, without explicit reference to the wall. This state is the so-called normal state and corresponds to the thermodynamic limit of the system [8]. Starting from a hydrodynamic description, it has recently been shown [9] that this macroscopic state exists in the bulk of a system confined between a vibrating wall and a steady one, at least in the limit of small gradients (and inelasticity). This was already implicit in the molecular dynamics results presented in ref. [6]. In this paper, we investigate the extension of the above state for higher inelasticities by means of the inelastic Boltzmann equation. 
The normal steady state we will consider is quite peculiar. A first point to realize is that it has no analogous in molecular systems. In the elastic limit, the gradients vanish and the state of the system becomes the usual thermodynamic equilibrium. For non-vanishing dissipation, the pressure of the gas and the temperature gradient are uniform, but they cannot take arbitrary values. Their ratio is determined by the degree of inelasticity of the collisions. This coupling between the gradient of a hydrodynamic field and the value of another (uniform) field is characteristic of granular gases, and a similar behaviour has been found for sheared systems $[10,11]$. The interest of this state is not purely formal, since it is the one observed in molecular dynamics simulations [6], in numerical solutions of the Boltzmann equation by means of the direct Monte Carlo simulation method [9], and qualitatively in experiments [12].

Although we have not been able to explicitly construct the solution of the Boltzmann equation for the state, some general features can be discussed. In particular, it will be shown to exhibit an algebraical tail for large velocities in the direction of decreasing temperature, indicating an overpopulation of particles with large velocities, as compared with the Gaussian distribution. This seems to be a quite general feature of granular systems [2].

The Boltzmann equation for the one-particle distribution function $f(\boldsymbol{r}, \boldsymbol{v}, t)$ of a dilute gas of smooth inelastic hard spheres $(d=3)$ or disks $(d=2)$ of mass $m$ and diameter $\sigma$ is

$$
\left(\partial_{t}+\boldsymbol{v} \cdot \nabla\right) f=\sigma^{d-1} \int \mathrm{d} \boldsymbol{v}_{1} \int \mathrm{d} \widehat{\boldsymbol{\sigma}} \theta(\boldsymbol{g} \cdot \widehat{\boldsymbol{\sigma}})(\boldsymbol{g} \cdot \widehat{\boldsymbol{\sigma}})\left(\alpha^{-2} b^{-1}-1\right) f(\boldsymbol{r}, \boldsymbol{v}, t) f\left(\boldsymbol{r}, \boldsymbol{v}_{1}, t\right),
$$

where $\boldsymbol{g}=\boldsymbol{v}-\boldsymbol{v}_{1}, \widehat{\boldsymbol{\sigma}}$ is a unit vector along the line of centers of the two colliding particles, $\theta$ is the Heaviside step function, and $b^{-1}$ is an operator transforming all the velocities $\boldsymbol{v}$ and $\boldsymbol{v}_{1}$ to its right into their precollisional values defined by momentum conservation and $\boldsymbol{g}^{\prime}=\boldsymbol{g}-(1+\alpha)(\boldsymbol{g} \cdot \widehat{\boldsymbol{\sigma}}) \widehat{\boldsymbol{\sigma}}$. Inelasticity in collisions is characterized through a constant coefficient of normal restitution $\alpha$, in the range $0<\alpha \leq 1$. The local number density $n(\boldsymbol{r}, t)$, flow velocity $\boldsymbol{u}(\boldsymbol{r}, t)$, and granular temperature $T(\boldsymbol{r}, t)$ are given by

$$
n=\int \mathrm{d} \boldsymbol{v} f, \quad n \boldsymbol{u}=\int \mathrm{d} \boldsymbol{v} \boldsymbol{v} f, \quad \frac{d}{2} n T=\frac{m}{2} \int \mathrm{d} \boldsymbol{v} V^{2} f,
$$

where $V(\boldsymbol{r}, t)=\boldsymbol{v}-\boldsymbol{u}$. Balance equations for these quantities are obtained by taking velocity moments in the Boltzmann equation (1),

$$
\begin{gathered}
\partial_{t} n+\nabla \cdot(n \boldsymbol{u})=0, \\
\partial_{t} \boldsymbol{u}+\boldsymbol{u} \cdot \nabla \boldsymbol{u}+(m n)^{-1} \nabla \cdot \mathrm{P}=0, \\
\partial_{t} T+\boldsymbol{u} \cdot \nabla T+2(d n)^{-1}(\mathrm{P}: \nabla \boldsymbol{u}+\nabla \cdot \boldsymbol{q})+T \zeta=0 .
\end{gathered}
$$

In the above equations the pressure tensor $\mathrm{P}$ and the heat flux $\boldsymbol{q}$ are the same functional of $f$ as for molecular fluids, $\mathrm{P}=m \int \mathrm{d} \boldsymbol{v} \boldsymbol{V} \boldsymbol{V} f, \boldsymbol{q}=\frac{m}{2} \int \mathrm{d} \boldsymbol{v} V^{2} \boldsymbol{V} f$, while the cooling rate $\zeta$, which vanishes in the elastic limit, is given by

$$
\zeta(\boldsymbol{r}, t)=\frac{m \pi^{\frac{d-1}{2}} \sigma^{d-1}\left(1-\alpha^{2}\right)}{4 d \Gamma\left(\frac{d+3}{2}\right) n T} \int \mathrm{d} \boldsymbol{v}_{1} \int \mathrm{d} \boldsymbol{v}_{2}\left|\boldsymbol{v}_{1}-\boldsymbol{v}_{2}\right|^{3} f\left(\boldsymbol{r}, \boldsymbol{v}_{1}, t\right) f\left(\boldsymbol{r}, \boldsymbol{v}_{2}, t\right) .
$$

We will search for a solution $f_{s}$ to the Boltzmann equation verifying the following conditions: a) it is time independent; b) there is no macroscopic flow velocity; c) there are gradients only in the direction of the $x$-axis; d) it scales in the form

$$
f_{s}(x, \boldsymbol{v})=n(x)\left[\frac{m}{2 T(x)}\right]^{d / 2} \varphi(\boldsymbol{\xi}), \quad \boldsymbol{\xi}=\left[\frac{m}{2 T(x)}\right]^{1 / 2} \boldsymbol{v} .
$$


This solution is similar to the one found by Goldshtein and Shapiro [13], describing the evolution of a homogeneous freely evolving granular fluid. Because of eq. (2), the function $\varphi$ must verify the conditions

$$
\int \mathrm{d} \boldsymbol{\xi} \varphi(\boldsymbol{\xi})=1, \quad \int \mathrm{d} \boldsymbol{\xi} \boldsymbol{\xi} \varphi(\boldsymbol{\xi})=0, \quad \int \mathrm{d} \boldsymbol{\xi} \xi^{2} \varphi(\boldsymbol{\xi})=\frac{d}{2} .
$$

For the state we are considering, eq. (3) becomes an identity, while eq. (4) together with eq. (7) imply that the pressure tensor is uniform. Finally, from the energy balance equation (5) we get

$$
\frac{1}{2} \frac{\mathrm{d} T}{\mathrm{~d} x} \int \mathrm{d} \boldsymbol{\xi} \xi^{2} \xi_{x} \varphi(\boldsymbol{\xi})=-\left(1-\alpha^{2}\right) \frac{\pi^{\frac{d-1}{2}} \sigma^{d-1} p}{4 \Gamma\left(\frac{d+3}{2}\right)} \int \mathrm{d} \boldsymbol{\xi}_{1} \int \mathrm{d} \boldsymbol{\xi}_{2}\left|\boldsymbol{\xi}_{1}-\boldsymbol{\xi}_{2}\right|^{3} \varphi\left(\boldsymbol{\xi}_{1}\right) \varphi\left(\boldsymbol{\xi}_{2}\right)
$$

where $p=d^{-1} \sum_{i} P_{i i}=n T$ is the constant pressure. Since the right-hand side of this equation does not depend on $x$, consistency requires that $\frac{\mathrm{d} T(x)}{\mathrm{d} x}=a=$ const, i.e. the temperature profile must be linear in $x$.

Equation (9) establishes a relationship between the temperature gradient and pressure that can be expressed in the form

$$
\frac{a}{p \sigma^{d-1}}=I[\varphi] \equiv-\frac{\left(1-\alpha^{2}\right) \pi^{\frac{d-1}{2}}}{2 \Gamma\left(\frac{d+3}{2}\right)} \frac{\int \mathrm{d} \boldsymbol{\xi}_{1} \int \mathrm{d} \boldsymbol{\xi}_{2}\left|\boldsymbol{\xi}_{1}-\boldsymbol{\xi}_{2}\right|^{3} \varphi\left(\boldsymbol{\xi}_{1}\right) \varphi\left(\boldsymbol{\xi}_{2}\right)}{\int \mathrm{d} \boldsymbol{\xi} \xi^{2} \xi_{x} \varphi(\boldsymbol{\xi})} .
$$

For symmetry considerations, the distribution function $f_{s}$ must be an even function of the velocity components perpendicular to the $x$-direction. This property, of course, is preserved by the Boltzmann equation. Then, in particular, there is no heat flux perpendicular to the temperature gradient. Substitution of eq. (7) into eq. (1) and use of eq. (10) leads to

$$
-I[\varphi]\left\{\xi_{x} \varphi(\boldsymbol{\xi})+\frac{1}{2} \xi_{x} \frac{\partial}{\partial \boldsymbol{\xi}} \cdot[\boldsymbol{\xi} \varphi(\boldsymbol{\xi})]\right\}=\int \mathrm{d} \boldsymbol{\xi}_{1} \int \mathrm{d} \widehat{\boldsymbol{\sigma}} \theta(\boldsymbol{\chi} \cdot \widehat{\boldsymbol{\sigma}})|\chi \cdot \widehat{\boldsymbol{\sigma}}|\left(\alpha^{-2} b^{-1}-1\right) \varphi(\boldsymbol{\xi}) \varphi\left(\boldsymbol{\xi}_{1}\right),
$$

where $\chi=\boldsymbol{\xi}-\boldsymbol{\xi}_{1}$. The structure of this equation is consistent with our assumption on the existence of a solution of the Boltzmann equation of the form (7). Then, $\varphi$ and also $I$ depend only on the coefficient of restitution $\alpha$ and eq. (10) implies that, for given $\alpha$, pressure and the gradient of temperature are not independent, but their ratio has a fixed value in the steady state we are considering. A similar behaviour has been found for the uniform shear flow state, for which the temperature and the shear rate are related $[10,11]$.

The component of the heat flux in the $x$-direction is

$$
q_{x}=\left(\frac{2 T(x)}{m}\right)^{1 / 2} p \int \mathrm{d} \boldsymbol{\xi} \xi^{2} \xi_{x} \varphi(\xi)
$$

Taking into account eq. (10) it follows that this flux is proportional to the temperature gradient $a$, namely it can be written in the form

$$
q_{x}=-\Phi(\alpha) \kappa_{0} a
$$

where $\Phi$ is an unknown function of the coefficient of restitution and $\kappa_{0}$ is the Boltzmann elastic heat conductivity. Therefore, we have obtained that the heat flux is linearly coupled to the temperature gradient. An approximated expression for $\Phi(\alpha)$ can be obtained by means 
of the Chapman-Enskog expansion. Using the results obtained in refs. [4] and [14] it is found that

$$
\Phi(\alpha)=\kappa^{*}(\alpha)-\mu^{*}(\alpha),
$$

where $\kappa^{*}(\alpha)$ and $\mu^{*}(\alpha)$ are functions only of the restitution coefficient and their explicit expressions can be found in $[9,14]$.

It is worth mentioning that for molecular systems $(\alpha=1)$ the Boltzmann equation also has an exact solution for Maxwell molecules, representing a steady state in which the Fourier law is obeyed for arbitrary temperature gradient [15].

There is no reason to expect that the distribution function $f_{s}$ has a simple dependence on the temperature gradient. Even more, calculations based on model kinetic equations for the nonlinear Boltzmann equation and applied to analogous states [16] seem to indicate that the distribution function has a quite complicated form. Nevertheless, from the results obtained in refs. [4] and [14], the expression of $f_{s}$ linearized in a, i.e. in the first Chapman-Enskog order, and evaluated in the first Sonine approximation can be written down. We will concentrate on the marginal velocity distribution $\varphi_{x}\left(\xi_{x}\right)=\int \mathrm{d} \boldsymbol{\xi}_{\perp} \varphi(\boldsymbol{\xi})$, where the integration is carried out over the $d-1$ components of $\boldsymbol{\xi}$ perpendicular to the $x$-axis. For this function it is found that

$$
\varphi_{x}\left(\xi_{x}\right)=\pi^{-1 / 2} e^{-\xi_{x}^{2}}\left\{1-\left[\frac{4 d^{2} \zeta^{*}\left(\kappa^{*}-\mu^{*}\right)}{(d+2)(d-1)}\right]^{1 / 2}\left(\xi_{x}^{2}-\frac{3}{2}\right) \xi_{x}\right\} .
$$

Upon deriving this expression, it has been assumed, without loss of generality, that $\partial T / \partial x=$ $a>0$. Otherwise, $\xi_{x}$ must be changed into $-\xi_{x}$. A factor of $a / p \sigma^{d-1}$ has been eliminated by using the Navier-Stokes equation for the temperature (see also eq. (10)), so that eq. (15) is written in a form which is consistent with eq. (11). We stress again that eq. (15) is, in principle, useful only in the small-gradient limit, that for the state we are dealing with is equivalent to small inelasticity. Moreover, it is restricted to values of $\left|\xi_{x}\right|$ of the order of unity. In fig. 1 we compare eq. (15) with numerical results obtained from direct Monte Carlo simulation of the Boltzmann equation of a vibrated system $[9,17]$ for $d=2$. Data from two different positions in the bulk of the system have been plotted (circles and squares). The relative difference of temperatures between both positions is about $30 \%$. The data overlap consistently with the scaling law in eq. (7). There is a quite good agreement with eq. (15) for small velocities and low dissipation, the deviations becoming more significant as the value of $\alpha$ decreases.

It is possible to obtain some information about the asymptotic form for high velocities of the solution of the Boltzmann equation by means of a method introduced by Krook and $\mathrm{Wu}[18]$ in the elastic case, and employed for homogeneous granular systems by Esipov and Pöschel [19] and by van Noije and Ernst [2]. From eq. (11) one obtains

$$
-I[\varphi]\left[\frac{3}{2} \xi_{x} \varphi_{x}\left(\xi_{x}\right)+\frac{1}{2} \xi_{x}^{2} \frac{\partial}{\partial \xi_{x}} \varphi_{x}\left(\xi_{x}\right)\right]=J_{x}^{(+)}[\varphi]-J_{x}^{(-)}[\varphi] .
$$

Here $J_{x}^{(+)}$and $J_{x}^{(-)}$denote the contributions from the gain and loss terms of the Boltzmann collision operator, respectively, i.e.

$$
J_{x}^{(-)}=\int \mathrm{d} \boldsymbol{\xi}_{\perp} \int \mathrm{d} \boldsymbol{\xi}_{1} \int \mathrm{d} \widehat{\boldsymbol{\sigma}} \theta(\boldsymbol{\chi} \cdot \widehat{\boldsymbol{\sigma}})|\chi \cdot \widehat{\boldsymbol{\sigma}}| \varphi(\boldsymbol{\xi}) \varphi\left(\boldsymbol{\xi}_{1}\right)
$$

and similarly $J_{x}^{(+)}$. Again, we assume that $a>0$, so that particles arriving at a given position with positive $\xi_{x}$ come from cooler regions. Therefore, particles with large and positive $\xi_{x}$ 

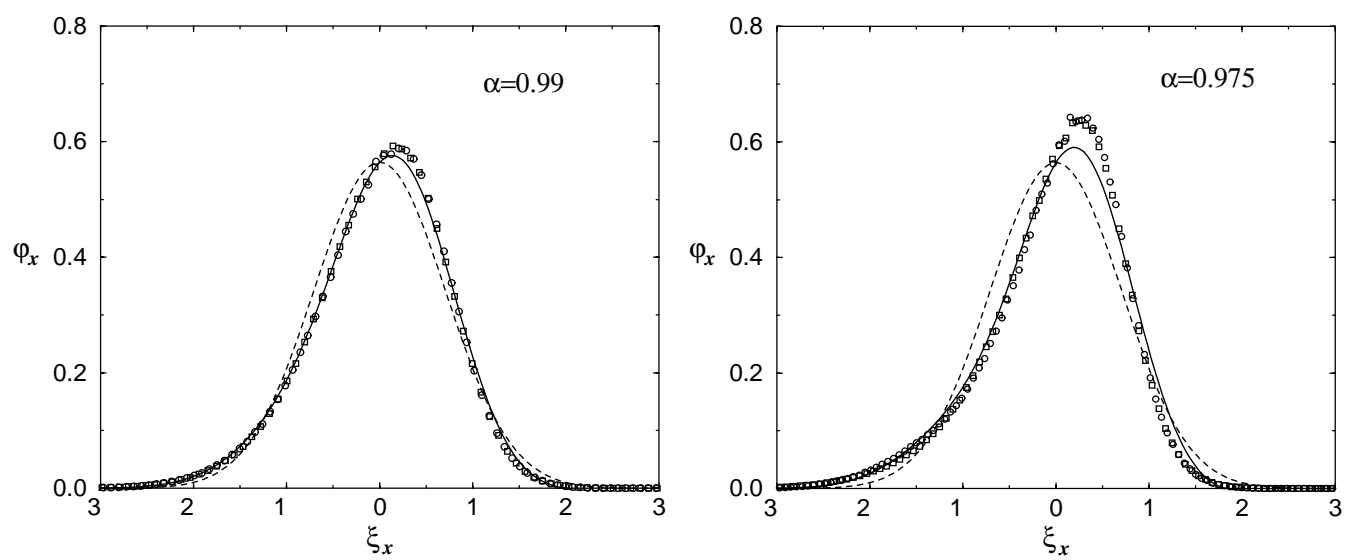

Fig. 1 - Marginal velocity distribution in the direction of the temperature gradient for $\alpha=0.99$ and $\alpha=0.975$ in a two-dimensional vibrated system. The continuous line is the theoretical prediction given in the main text, the symbols are from direct Monte Carlo simulation of the Boltzmann equation and correspond to two different positions (squares and circles) in the bulk, and the dotted line is the Gaussian, plotted for reference.

at a given location are mostly generated as an effect of collisions with other particles in the same region. On the other hand, particles with large $\left|\xi_{x}\right|$ but $\xi_{x}<0$ might come from regions corresponding to higher temperatures and the probability they are generated as an effect of collisions at the given location is low. For the latter particles, i.e. those with $\xi_{x}<0$ and $\left|\xi_{x}\right| \gg 1$, the gain term is negligible as compared with the loss one. The dominant contribution to the collision integral corresponds to collisions where the velocity of particle 1 is typically in the thermal region, so that $|\chi|$ can be replaced by $|\boldsymbol{\xi}|$. Since the same kind of reasoning can be applied to the integral over the normal component $\boldsymbol{\xi}_{\perp},|\boldsymbol{\xi}|$ can in turn be replaced by $\left|\xi_{x}\right|$. Equation (17) can be approximated by

$$
J_{x}^{(-)} \approx \frac{\pi^{\frac{d-1}{2}}}{\Gamma\left(\frac{d+1}{2}\right)}\left|\xi_{x}\right| \varphi_{x}\left(\xi_{x}\right),
$$

so that eq. (16) can be simplified to

$$
-I[\varphi]\left(\frac{3}{2} \xi_{x} \varphi_{x}\left(\xi_{x}\right)+\frac{1}{2} \xi_{x}^{2} \frac{\partial}{\partial \xi_{x}} \varphi_{x}\left(\xi_{x}\right)\right)=\frac{\pi^{\frac{d-1}{2}}}{\Gamma\left(\frac{d+1}{2}\right)} \xi_{x} \varphi_{x}\left(\xi_{x}\right),
$$

whose solution has the form

$$
\varphi_{x}\left(\xi_{x}\right)=\mathcal{A}\left|\xi_{x}\right|^{-b(\alpha)},
$$

where $\mathcal{A}$ is an undetermined integration constant and

$$
b(\alpha)=\frac{2 \pi^{\frac{d-1}{2}}}{\Gamma\left(\frac{d+1}{2}\right) I}+3 .
$$

Note that for $a>0$, it is $I[\varphi]>0$, as is seen from eq. (10). In the same first Sonine approximation used to derive eq. (14), one obtains

$$
I=\left[\frac{32(d-1) \pi^{d-1}\left(1-\alpha^{2}\right)}{d(d+2)^{2} \Gamma(d / 2)^{2}\left(\kappa^{*}-\mu^{*}\right)}\right]^{1 / 2} .
$$


Then, we have obtained that for large component of the velocity in the opposite direction to the increase of the temperature, there is an algebraic tail in the marginal velocity distribution. This corresponds to an overpopulation for those velocities when compared with the Gaussian distribution. As already mentioned, this seems to be a quite general feature of systems of particles with dissipative interactions.

Since in most of the previous theoretical analysis of velocity distribution functions for granular gases, an exponential rather than algebraical decay has been found [2], it could be argued whether the result presented here is an artifact of the asymptotic analysis we have carried out. Nevertheless, a careful inspection of each of the steps leading to eq. (20) shows that it represents in any case the fastest possible decay of the marginal velocity distribution for large negative values of $\xi_{x}$. Therefore, in particular, such decay cannot be exponential. Of course, there is no contradiction here, as the previous studies correspond to different physical situations, in which the system was either evolving freely or driven by a stochastic force acting on all the particles. Moreover, the algebraical decay is compatible with the existence of the first moments of the velocity distribution, since the exponent of the power law is always larger that three and it is expected to grow very fast as $\alpha$ approaches unity (see eq. (10)). For large component of the velocity in the direction of positive gradient of the temperature, i.e. $\xi_{x} \gg 1$, the dominant part of the collision integral is the gain term $J_{x}^{(+)}$, and the reasoning developed above does not apply.

This research was partially supported by Grant No. PB98-1124 from the Dirección General de Investigación Científica y Técnica (Spain).

\section{REFERENCES}

[1] Haff P. K., J. Fluid Mech., 134 (1983) 401; Campbell C. S., Annu. Rev. Fluid Mech., 22 (1990) 57.

[2] VAn Noije T. P. C. and Ernst M. H., Granular Matter, 1 (1998) 57.

[3] Sela N. and Goldhirsch I., J. Fluid Mech., 361 (1998) 41.

[4] Brey J. J., Dufty J. W., Kim C. S. and Santos A., Phys. Rev. E, 58 (1998) 4638.

[5] Sela N., Goldhirsch I. and Noskowitz S. N., Phys. Fluids, 8 (1996) 2337.

[6] Grossman E. L., Zhou T. and Ben-Naim E., Phys. Rev. E, 55 (1997) 2846.

[7] Brey J. J. and Cubero D., Phys. Rev. E, 57 (1998) 2019.

[8] Balecu R., Equilibrium and Non-equilibrium Statistical Mechanics (Wiley-Interscience, New York) 1976.

[9] Brey J. J., Ruiz-Montero M. J. and Moreno F., Phys. Rev. E, 62 (2000) 5339.

[10] Jenkins J. T. and Richman M. W., J. Fluid Mech., 192 (1988) 313.

[11] Goldhirsch I. and Tan M. L., Phys. Fluids, 8 (1996) 1752.

[12] Kudrolli A., Wolpert M. and Gollub J. P., Phys. Rev. Lett., 78 (1997) 1383.

[13] Goldshtein A. and Shapiro M., J. Fluid Mech., 282 (1995) 75.

[14] Brey J. J. and Cubero D., in Granular Gases, edited by S. Luding and T. Pöschel, to be published in Lect. Notes Phys. (Springer-Verlag, Berlin).

[15] Asmolov E. S., Makashev N. K. and Nosik V. I., Dokl. Akad. Nauk SSSR, 249 (1979) 577 (Sov. Phys. Dokl., 24 (1979) 892).

[16] Brey J. J., Santos A. and Dufty J. W., Phys. Rev. A, 36 (1987) 2842.

[17] BIRD G., Molecular Gas Dynamics and the Direct Simulation of Gas Flows (Clarendon Press, Oxford) 1994.

[18] Krook M. and Wu T. T., Phys. Rev. Lett., 36 (1975) 1107.

[19] Esipov S. E. and Pöschel T., J. Stat. Phys., 86 (1997) 1385. 